doi: $10.13108 / 2015-7-1-70$

UDC 517.984

\title{
GENERAL REGULARIZED TRACE FORMULAE FOR LOADED EQUATIONS
}

\section{I.D. TSOPANOV}

\begin{abstract}
We consider regularized traces for differential operators with the coefficients at the powers of a spectral parameter being the values of an unknown function at prescribed points in its domain. Such differential operators are interpreted as polynomial operator pencils whose coefficients are unbounded fininte-dimensional operators. Basing on the theory of M.V. Keldysh, we construct general regularized trace formulae for such operator pencils. The obtained formulae develop a known result by V.A. Sadovnichii and V.A. Lyubishkin for relative finite-dimensional perturbations of self-adjoint operators.
\end{abstract}

Keywords: spectrum, operator pencil, regularized traces.

Mathematics Subject Classification: 47A55, 34B07, 34L15

\section{INTRODUCTION}

We consider the operator pencil

$$
N_{\lambda}=A-Q_{0}-\lambda Q_{1}-\cdots-\lambda^{n-1} Q_{n-1}-\lambda^{n} E,
$$

where $A$ is an unbounded self-adjoint operator in a separable Hilbert space $\mathfrak{H}$ with a compact resolvent. Operators $Q_{0}, Q_{1}, \ldots, Q_{n-1}$ are assumed to be $A$-finite-dimensional, i.e., they read as $Q_{j}=P_{j} A$, where $P_{j}$ are finite-dimensional bounded operators in $\mathfrak{H}$ :

$$
\forall h \in \mathfrak{H} \quad P_{j} h=\sum_{l=1}^{n_{j}}\left(h, \varphi_{l}^{j}\right) \psi_{l}^{j},
$$

where $\varphi_{l}^{j}, \psi_{l}^{j} \in \mathfrak{H}, j=0,1, \ldots, n-1 ; l=1, \ldots, n_{j}$. We observe that if vectors $\varphi_{l}^{j}$ do not belong to the domain of operator $A$, then $Q_{j}$ is an unbounded operator in $\mathfrak{H}$.

The above operator pencils appear, for instance, while solving initial boundary value problems for loaded equations [1, 2]

$$
\frac{\partial^{2} u(t, x)}{\partial^{2} t}=\frac{\partial^{2} u(t, x)}{\partial^{2} x}+\sum_{i=1}^{\nu} a_{i}(x) u\left(t, x_{i}\right)+\sum_{j=1}^{\mu} b_{j}(x) \frac{\partial u\left(t, z_{j}\right)}{\partial t} .
$$

by the Fourier method.

As a regularized trace formula for pencil (1) we call the formula

$$
\sum_{\nu}\left(\mu_{\nu}^{s}-\eta_{\nu}^{s}-c_{\nu}(s)\right)=F(s)
$$

where $\mu_{\nu}$ and $\eta_{\nu}$ are the eigenvalues of pencils $N_{\lambda}$ and $A_{\lambda^{n}} \stackrel{\text { def }}{=} A-\lambda^{n} E$, respectively, $s$ is the arc length, $c_{\nu}(s)$ and $F(s)$ are some quantities. In the left hand side of (3) the sum symbol denotes a summation, probably with some brackets, over all eigenvalues of pencils $N_{\lambda}$ and $A_{\lambda^{n}}$, and the way of placing brackets depend on the behavior of spectrum of operator $A$.

I.D. Tsopanov, General Regularized trace Formulae FOR LOADED EQUations.

(C) TsOpanov I.D. 2015.

Submitted October 13, 2014. 
As $n=1$ and $s=1$, a regularized trace formula was obtained first in work [3] for a relative finite-dimensional perturbation of an unbounded self-adjoint operator under rather general assumptions for the sparseness of its spectrum. In work [4] there were obtained regularized traces as $n=1$ and $s>1$ for relative finite-dimensional perturbation and they are expressed as recurrent formulae. As $s>1$, the construction of regularized trace formulae in the case of infinite-dimensional perturbations is a more complicated problem. By the methods of the perturbation theory for abstract operators with discrete spectra in Hilbert space, formulae (3) were obtained in [5] (see also [6]) under a condition for the sparseness of the spectrum of an unperturbed operator. An essential progress in this direction was made in [7], where the restrictions for the sparseness of the spectrum were omitted. The survey and detailed analysis of the results obtained in the theory of regularized traces of operators were provided in [8].

Paper [9] was likely the first work devoted to constructing regularized trace formulae by analytic methods for a loaded ordinary differential equation, which in some cases can be treated as operator pencil (1). In the present work we obtain regularized trace formulae (3) for operator pencils (1) and arbitrary $s \in \mathbb{N}$.

It is interesting to note that the history of regularized traces for polynomial operator pencils reproduce the history of operator traces. Works [10]-[14] are devoted to constructing the formulae for the sums of the inverses for the eigenvalues of polynomial operator pencils. The main approach in these works is the linearization method and the known Lidskii's theorem on trace of a nuclear operator [15].

\section{PRELIMINARIES}

In what follows we assume that $\lambda=0 \notin \sigma(A)$, i.e., $T=A^{-1}$ is a compact operator. From original pencil (1) we pass to the pencil $L_{\lambda}=N_{\lambda} A^{-1}$ :

$$
L_{\lambda}=E-P_{0}-\lambda P_{1}-\cdots-\lambda^{n-1} P_{n-1}-\lambda^{n} T .
$$

A complex number $\mu$ is an eigenvalue of pencil $L_{\lambda}$ if $L_{\mu} y=0$ for some non-zero vector $y \in \mathfrak{H}$. It was shown in work [16] that the spectrum of pencil $L_{\lambda}$ is formed by a discrete set of eigenvalues $\sigma\left(L_{\lambda}\right)=\left\{\mu_{k}\right\}_{k=1}^{\infty}$ with the only accumulation point at infinity.

Let $\left\{\lambda_{\nu}\right\}_{\nu=1}^{\infty}$ be the eigenvalues of pencil $T_{\lambda}=E-\lambda T$, i.e., $\sigma\left(T_{\lambda}\right)=\left\{\lambda_{\nu}\right\}_{\nu=1}^{\infty}$. We consider also the pencil $T_{\lambda^{n}} \stackrel{\text { def }}{=} E-\lambda^{n} T$ whose eigenvalues are denoted by $\eta_{k}$, i.e., $\sigma\left(T_{\lambda^{n}}\right)=\left\{\eta_{k}\right\}_{k=1}^{\infty}$. We index the eigenvalues in the order of ascending absolute values with the multiplicities taken into account. We have the following lemma [16]

Lemma 1 (M.V. Keldysh). Let $E-L_{\lambda}$ be an analytic in $\mathfrak{D} \subseteq \mathbb{C}$ operator function with the values in the ideal $\mathfrak{S}_{\infty}$ of compact operators. Then the trace of the principal part of the operator $\frac{\partial L_{\lambda}}{\partial \lambda} L_{\lambda}^{-1}$ for the pole $\lambda=c$ is equal to $\frac{N}{\lambda-c}$, where $N$ is an algebraic multiplicity of eigenvalue $\lambda=c$ of pencil $L_{\lambda}$.

If we denote by $\left[\frac{\partial L_{\lambda}}{\partial \lambda} L_{\lambda}^{-1}\right]$ the principal part of operator $\frac{\partial L_{\lambda}}{\partial \lambda} L_{\lambda}^{-1}$, and by $\operatorname{Tr}(\bullet)$ we denote the trace, Lemma (1) implies the relation

$$
\frac{1}{2 \pi i} \oint_{\Gamma_{c}} \lambda^{s} \operatorname{Tr}\left(\left[\frac{\partial L_{\lambda}}{\partial \lambda} L_{\lambda}^{-1}\right]\right) d \lambda=N c^{s},
$$

where $\Gamma_{c}$ is a circle of a sufficiently small radius centered $\lambda=c$ passed counterclockwise.

\section{PREliminary REgUlarized trace Formula}

Suppose that the counting function of characteristic values of operator $T=A^{-1}$ satisfies the condition

$$
\varliminf_{r \rightarrow \infty} \frac{N(r)}{r^{\alpha}}=\varepsilon<\infty \quad \text { as } \quad 0<\alpha \leqslant \frac{1}{n}
$$


where $\varepsilon$ is a positive constant (i.e., $0<\varepsilon \leqslant \infty)$. We introduce the notations: $r_{k}=\left|\lambda_{k}^{1 / n}\right|$, $d_{k}=r_{k+1}-r_{k}$. The proof of the next lemma was provided in [17].

Lemma 2. Under condition (6) for function $N(\lambda)$, there exists a subsequence of the natural series $\left\{k_{\nu}\right\}_{\nu=1}^{\infty}$ such that $d_{k_{\nu}}=r_{k_{\nu}+1}-r_{k_{\nu}} \geqslant \varepsilon_{0} \forall \nu \in \mathbb{N}$, where $\varepsilon_{0}>0$ is a constant.

Corollary 1. There exists an infinite system of expanding concentric circles $\left\{\Gamma_{\nu}\right\}_{\nu=1}^{\infty}$ centered at the origin and containing no spectrum of pencil $T_{\lambda^{n}}$ such that the distance $\delta_{\nu}$ from circle $\Gamma_{\nu}$ to spectrum $\sigma\left(T_{\lambda^{n}}\right)$ satisfies the condition $\delta_{\nu} \geqslant \varepsilon_{0} / 2 \forall \nu \in \mathbb{N}$.

Доказательство. As $\Gamma_{\nu}$ we choose the circle of radius $\widetilde{R}_{\nu}=r_{k_{\nu}}+\frac{1}{2} d_{k_{\nu}}$ centered at the origin. Then $\Gamma_{\nu}$ contains no spectrum $\sigma\left(T_{\lambda^{n}}\right)$, i.e., points in $\sigma\left(T_{\lambda^{n}}\right)$ are located on circumferences of radii $r_{k}, k \in \mathbb{N}$, centered at the origin. Moreover, since the points in spectrum $\sigma\left(T_{\lambda^{n}}\right)$ are located at the rays $\arg \lambda=\frac{k \pi}{n}, k=0,1, \ldots, 2 n-1$, then $\delta_{\nu} \geqslant d_{k_{\nu}} / 2$. Hence, in accordance with Lemma 2, $\delta_{\nu} \geqslant \varepsilon_{0} / 2 \forall \nu \in \mathbb{N}$.

Lemma 3. Let $P$ be a finite-dimensional operator in $\mathfrak{H}: P=\sum_{l=1}^{t}\left(\bullet, \varphi_{l}\right) \psi_{l}, \psi_{l} \in \mathfrak{D}\left(T^{-1}\right)$, $l=1,2, \ldots, t$. Then for $j=0,1,2, \ldots, n-1$ and $R_{\lambda}=\left(E-\lambda^{n} T\right)^{-1}$ the relations $\left\|\lambda^{j} R_{\lambda} P\right\| \rightarrow 0$ hold true as $\lambda \in \Gamma_{\nu}$ and $\nu \rightarrow \infty$ uniformly in $\arg \lambda$. (see [17]).

By means of Lemma 3 we can represent operator function $L_{\lambda}^{-1}$ as a series.

Corollary 2. For $\lambda \in \Gamma_{\nu}$ and sufficiently large $\nu$ the formula

$$
L_{\lambda}^{-1}=\sum_{k=0}^{\infty}\left\{R_{\lambda} \sum_{j=0}^{n-1} \lambda^{j} P_{j}\right\}^{k} R_{\lambda}
$$

holds true, where the series converges in the operator topology uniformly in $\arg \lambda$.

Multiplying the left and right hand sides of this identity by the left and right hand sides of the identity $\frac{\partial L_{\lambda}}{\partial \lambda}=-\sum_{j=0}^{n-1} j \lambda^{j-1} P_{j}-n \lambda^{n-1} T$, we obtain

$$
\begin{aligned}
\frac{\partial L_{\lambda}}{\partial \lambda} L_{\lambda}^{-1} & +n \lambda^{n-1} T R_{\lambda}=-\sum_{j=1}^{n-1} j \lambda^{j-1} P_{j} R_{\lambda} \\
& -\sum_{j=1}^{n-1} j \lambda^{j-1} P_{j} \sum_{k=1}^{\infty}\left\{R_{\lambda} \sum_{l=0}^{n-1} \lambda^{l} P_{l}\right\}^{k} R_{\lambda}-n \lambda^{n-1} T \sum_{k=1}^{\infty}\left\{R_{\lambda} \sum_{l=0}^{n-1} \lambda^{l} P_{l}\right\}^{k} R_{\lambda} .
\end{aligned}
$$

Integrating identity (8) over the contour $\Gamma_{\nu}, \nu \geqslant m_{0}$, by formula (5) with $s=0$, in the left hand side we obtain:

$$
\begin{aligned}
& \frac{1}{2 \pi i} \oint_{\Gamma_{\nu}} \operatorname{Tr}\left(\frac{\partial L_{\lambda}}{\partial \lambda} L_{\lambda}^{-1}+n \lambda^{n-1} T R_{\lambda}\right) d \lambda=\frac{1}{2 \pi i} \oint_{\Gamma_{\nu}} \operatorname{Tr}\left(\left[\frac{\partial L_{\lambda}}{\partial \lambda} L_{\lambda}^{-1}\right]\right) d \lambda+ \\
& \quad+\frac{1}{2 \pi i} \oint_{\Gamma_{\nu}} \operatorname{Tr}\left(\left[n \lambda^{n-1} T R_{\lambda}\right]\right) d \lambda=M_{\nu}-N_{\nu}
\end{aligned}
$$

Here $M_{\nu}$ and $N_{\nu}$ are numbers of the eigenvalues taken counting multiplicities of pencils $L_{\lambda}$ and $T_{\lambda^{n}}$ lying respectively inside contour $\Gamma_{\nu}$.

Lemma 4. The left hand side of identity (9) tends to zero as $\nu \rightarrow \infty$. Since $N_{\nu}$ and $M_{\nu}$ are natural numbers, there exists an index $m_{0}$ such that for $\nu \geqslant m_{0}$ we have $M_{\nu}=N_{\nu}$, i.e., starting from some index $m_{0}$, all circles $\Gamma_{\nu}$ contain the same number (counting multiplicities) of the eigenvalues of pencils $L_{\lambda}$ and $T_{\lambda^{n}}$. 
The proof will be given later once we study function $F(s)$ defined by identity (11).

In what follows we assume that index $m_{0}$ is such that inequality $\nu \geqslant m_{0}$ ensures all the aforementioned conditions. It follows from Lemma 4 that as $\nu \geqslant m_{0}$, between contours $\Gamma_{m+1}$ and $\Gamma_{m}$ there is the same number (counting multiplicities) of the eigenvalues of pencils $L_{\lambda}$ and $T_{\lambda^{n}}$, namely $N_{m+1}-N_{m}$ eigenvalues. Hence, multiplying $(8)$ by $\lambda^{s}(2 \pi i)^{-1}$, calculating the trace, integrating over contour $\Gamma_{\nu}$, passing to the limit $\nu \rightarrow \infty$ by formula (5), in the left hand side we obtain:

$$
\lim _{\nu \rightarrow \infty} \sum_{k=1}^{N_{\nu}}\left(\mu_{k}^{s}-\eta_{k}^{s}\right)=\left(\sum_{k=1}^{N_{m_{0}}}+\sum_{\nu=m_{0}}^{\infty} \sum_{k=N_{\nu}+1}^{N_{\nu+1}}\right)\left(\mu_{k}^{s}-\eta_{k}^{s}\right) .
$$

In order to get formula (3), we need to study the right hand side of (8) after the above described procedure, namely, the expression:

$$
\begin{aligned}
F(s)=-\lim _{\nu \rightarrow \infty}\{ & \sum_{j=1}^{n-1} \frac{j}{2 \pi i} \oint_{\Gamma_{\nu}} \lambda^{j+s-1} \operatorname{Tr}\left(P_{j} R_{\lambda}\right) d \lambda \\
& +\sum_{j=1}^{n-1} \frac{j}{2 \pi i} \oint_{\Gamma_{\nu}} \operatorname{Tr}\left(\sum_{k=1}^{\infty} \lambda^{j+s-1} P_{j}\left(R_{\lambda} \sum_{l=0}^{n-1} \lambda^{l} P_{l}\right)^{k} R_{\lambda}\right) d \lambda \\
& \left.+\frac{n}{2 \pi i} \oint_{\Gamma_{\nu}} \operatorname{Tr}\left(\sum_{k=1}^{\infty} \lambda^{n+s-1} T\left(R_{\lambda} \sum_{l=0}^{n-1} \lambda^{l} P_{l}\right)^{k} R_{\lambda}\right) d \lambda\right\} .
\end{aligned}
$$

We have

$$
\left(\sum_{l=0}^{n-1} \lambda^{l} R_{\lambda} P_{l}\right)^{k}=\left(R_{\lambda} P_{0}\right)^{k}+\sum_{m=1}^{k(n-1)} \lambda^{m} \sum_{\substack{\alpha_{1}+\alpha_{2}+\ldots+\alpha_{k}=m \\ 0 \leqslant \alpha_{1}, \ldots, \alpha_{k} \leqslant n-1}} R_{\lambda} P_{\alpha_{1}} \cdots R_{\lambda} P_{\alpha_{k}}
$$

Denoting the internal sum by $\sum_{m} P_{\alpha_{1} \ldots \alpha_{k}}$, we arrive at

$$
\left(\sum_{l=0}^{n-1} \lambda^{l} R_{\lambda} P_{l}\right)^{k}=\left(R_{\lambda} P_{0}\right)^{k}+\sum_{m=1}^{k(n-1)} \lambda^{m} \sum_{m} P_{\alpha_{1} \ldots \alpha_{k}} .
$$

Employing identity (12), by 11 we obtain:

$$
\begin{aligned}
F(s)=-\lim _{\nu \rightarrow \infty}\{ & \sum_{j=1}^{n-1} \frac{j}{2 \pi i} \oint_{\Gamma_{\nu}} \lambda^{j+s-1} \operatorname{Tr}\left(R_{\lambda} P_{j}\right) d \lambda \\
& +\sum_{j=1}^{n-1} \sum_{k=1}^{\infty} \frac{j}{2 \pi i} \oint_{\Gamma_{\nu}} \lambda^{j+s-1} \operatorname{Tr}\left(R_{\lambda} P_{j}\left(R_{\lambda} P_{0}\right)^{k}\right) d \lambda \\
& +\sum_{j=1}^{n-1} \sum_{k=1}^{\infty} \sum_{m=1}^{k(n-1)} \frac{j}{2 \pi i} \oint_{\Gamma_{\nu}} \lambda^{j+s+m-1} \operatorname{Tr}\left(R_{\lambda} P_{j} \sum_{m} P_{\alpha_{1} \ldots \alpha_{k}}\right) d \lambda \\
& +\sum_{k=1}^{\infty} \frac{n}{2 \pi i} \oint_{\Gamma_{\nu}} \lambda^{n+s-1} \operatorname{Tr}\left(T R_{\lambda}\left(R_{\lambda} P_{0}\right)^{k}\right) d \lambda \\
& \left.+\sum_{k=1}^{\infty} \sum_{m=1}^{k(n-1)} \frac{n}{2 \pi i} \oint_{\Gamma_{\nu}} \lambda^{m+n+s-1} \operatorname{Tr}\left(R_{\lambda} T \sum_{m} P_{\alpha_{1} \ldots \alpha_{k}}\right) d \lambda\right\} .
\end{aligned}
$$


We denote by $J_{l}^{\nu}(s)(l=1,2, \ldots, 5)$ in the right hand side of $(13)$, respectively. Our next step is to calculate $\lim _{\nu \rightarrow \infty} J_{l}^{\nu}(s) \forall s \in \mathbb{N} \cup\{0\} l=1,2, \ldots, 5$. In order to do it, we need some preliminary formulae to which the next section is devoted.

We note that the subsequent considerations imply easily the well-definiteness of the passage to the limit as $\nu \rightarrow \infty$ in infinite series in 13 .

\section{Auxiliary FOrmulae}

We recall that $T$ is a compact self-adjoint operator in separable Hilbert space $\mathfrak{H}$ and its counting function satisfies condition (6). Let $\left\{e_{j}\right\}_{j=1}^{\infty}$ be the orthonormalized basis in space $\mathfrak{H}$ formed by the eigenvectors of operator $T$. In what follows by $n$ we denote the orders of pencils (1), (4).

4.1. Calculations with $P_{\lambda}^{k}$. We employ the system of circles $\left\{\Gamma_{\nu}\right\}_{\nu=1}^{\infty}$ constructed in Corollary 1. Let $N \geqslant 0$ be an integer number and $P$ be a finite-dimensional operator and $\psi_{l} \in \mathfrak{D}\left(T^{-(N+1)}\right), l=1, \ldots, t$. Employing then the identity $R_{\lambda} e_{j}=\lambda_{j}\left(\lambda_{j}-\lambda^{n}\right)^{-1} e_{j}$, we obtain

$$
\operatorname{Tr}\left(R_{\lambda} P\right)=\sum_{l=1}^{t}\left(R_{\lambda} \psi_{l}, \varphi_{l}\right)=\sum_{l=1}^{t} \sum_{k=1}^{\infty} \frac{\lambda_{k}\left(\psi_{l}, e_{k}\right)\left(e_{k}, \varphi_{l}\right)}{\lambda_{k}-\lambda^{n}} .
$$

In the right hand side we apply the identity

$$
\frac{1}{\lambda_{k}-\lambda^{n}}=-\frac{1}{\lambda^{n}}+\frac{\lambda_{k}}{\lambda^{n}\left(\lambda_{k}-\lambda^{n}\right)}
$$

$N$ times. It leads us to the formula

$$
\operatorname{Tr}\left(R_{\lambda} P\right)=-\sum_{l=1}^{t} \sum_{k=1}^{N} \frac{\left(T^{-k} \psi_{l}, \varphi_{l}\right)}{\lambda^{n k}}+\sum_{l=1}^{t} \sum_{k=1}^{\infty} \frac{\lambda_{k}^{N+1}\left(\psi_{l}, e_{k}\right)\left(e_{k}, \varphi_{l}\right)}{\lambda^{n N}\left(\lambda_{k}-\lambda^{n}\right)} .
$$

We denote the second term by $\Phi_{N}(\lambda)$. Employing then the trick used in the proof of Lemma 3 , it is easy to show that as $\psi_{l} \in \mathfrak{D}\left(T^{-(N+1)}\right), l=1,2, \ldots, t$, and $\lambda \in \Gamma_{\nu}$ the identity $\Phi_{N}(\lambda)=$ $o\left(\lambda^{-n N}\right), \nu \rightarrow \infty$, holds true and this identity is uniform in $\arg \lambda$.

Suppose that we have set (2) of finite-dimensional operators. Let $P_{l}^{j}=\left(\bullet, \varphi_{l}^{j}\right) \psi_{l}^{j}$, then $P_{j}=$ $\sum_{l=1}^{n_{j}} P_{l}^{j}$. We consider the operator function $P_{\lambda}^{k} \stackrel{\text { def }}{=} R_{\lambda} P_{1} R_{\lambda} P_{2} \cdots R_{\lambda} P_{k}$. It is easy to obtain that

$$
\begin{gathered}
P_{\lambda}^{k}=\sum_{\substack{l_{1}, l_{2}, \ldots, l_{k} \\
0 \leqslant l_{j} \leqslant n_{j}}} R_{\lambda} P_{l_{1}}^{1} R_{\lambda} P_{l_{2}}^{2} \cdots R_{\lambda} P_{l_{k}}^{k}, \\
\operatorname{Tr}\left(P_{\lambda}^{k}\right)=\sum_{\substack{l_{1}, l_{2}, \ldots, l_{k} \\
0 \leqslant l_{j} \leqslant n_{j}}}\left\{\prod_{j=1}^{k-1}\left(R_{\lambda} \psi_{l_{j+1}}^{j+1}, \varphi_{l_{j}}^{j}\right)\right\}\left(R_{\lambda} \psi_{l_{1}}^{1}, \varphi_{l_{k}}^{k}\right) .
\end{gathered}
$$

Suppose that

$$
\psi_{l}^{j} \in \mathfrak{D}\left(T^{-(N+2)}\right), \quad N \in \mathbb{N}_{0}, \quad j=1,2, \ldots, k ; \quad l=1, \ldots, n_{j} .
$$

Hence, by relations (15) and (16), as $\lambda \in \Gamma_{\nu}, \nu \rightarrow \infty$ we get

$$
\begin{aligned}
\operatorname{Tr}\left(P_{\lambda}^{k}\right)= & \sum_{\substack{l_{1}, l_{2}, \ldots, l_{k} \\
0 \leqslant l_{j} \leqslant n_{j}}}(-1)^{k} \sum_{\substack{p_{1}+p_{2}+\ldots+p_{k}=M \\
p_{1}, \ldots, p_{k} \geqslant 1}} \lambda^{-M n}\left\{\prod_{j=1}^{k-1}\left(T^{-p_{j+1}} \psi_{l_{j+1}}^{j+1}, \varphi_{l_{j}}^{j}\right)\right\}\left(T^{-p_{1}} \psi_{l_{1}}^{1}, \varphi_{l_{k}}^{k}\right) \\
& +B_{\lambda}^{M}+o\left(\lambda^{-n(N+k)}\right)
\end{aligned}
$$


where natural $M$ satisfies the condition $k \leqslant M \leqslant N+k$, and the symbol $B_{\lambda}^{M}$ stands for the sum of terms obeying $p_{1}+p_{2}+\ldots+p_{k} \neq M$.

It follows from (18) that for each natural $M$ satisfying the inequalities $k \leqslant M \leqslant N+k$, the identity

$$
\begin{aligned}
\lim _{\nu \rightarrow \infty} \frac{1}{2 \pi i} \oint_{\Gamma_{\nu}} \lambda^{M n-1} \operatorname{Tr}\left(P_{\lambda}^{k}\right) d \lambda & =\sum_{\substack{l_{1}, l_{2}, \ldots, l_{k} \\
0 \leqslant l_{j} \leqslant n_{j}}}(-1)^{k} \sum_{\substack{p_{1}+p_{2}+\ldots+p_{k}=M \\
p_{1}, \ldots, p_{k} \geqslant 1}}\left\{\prod_{j=1}^{k-1}\left(T^{-p_{j+1}} \psi_{l_{j+1}}^{j+1}, \varphi_{l_{j}}^{j}\right)\right\}\left(T^{-p_{1}} \psi_{l_{1}}^{1}, \varphi_{l_{k}}^{k}\right) \\
& =(-1)^{k} \sum_{\substack{p_{1}+p_{2}+\ldots+p_{k}=M \\
p_{1}, \ldots, p_{k} \geqslant 1}} \operatorname{Tr}\left(T^{-p_{1}} P_{1} \cdots T^{-p_{k}} P_{k}\right)
\end{aligned}
$$

holds true. Thus, we arrive at

Lemma 5. Suppose that we are given finite-dimensional operators $P_{1}, \ldots, P_{k}$ in Hilbert space $\mathfrak{H}$ and condition (17) is satisfied. If natural number $M$ is such that $k \leqslant M \leqslant N+k$, then operator function $P_{\lambda}^{k} \stackrel{\text { def }}{=} R_{\lambda} P_{1} R_{\lambda} P_{2} \cdots R_{\lambda} P_{k}$ satisfies (19). If in the left hand side of (19) we replace $M n$ by some natural $s: k n<s<(N+k) n$ not being a multiple of $n$, it vanishes.

4.2. Calculations with $Q_{\lambda}^{k}$. In what follows we shall make similar relations for operator function $Q_{\lambda}^{k} \stackrel{\text { def }}{=} R_{\lambda}^{2} P_{1} R_{\lambda} P_{2} \cdots R_{\lambda} P_{k}$.

Applying identity (14), as above, we obtain the identity

$$
\operatorname{Tr}\left(R_{\lambda}^{2} P\right)=\sum_{l=1}^{t} \sum_{j=2}^{N+1}(j-1) \frac{\left(T^{-j} \psi_{l}, \varphi_{l}\right)}{\lambda^{n j}}+\widetilde{F}_{\lambda},
$$

where $P$ is a finite-dimensional operator satisfying $\psi_{l} \in \mathfrak{D}\left(T^{-(N+2)}\right), l=1,2, \ldots, t$,

$$
\begin{aligned}
& \widetilde{F}_{\lambda}=F_{\lambda}^{1}+F_{\lambda}^{2}+F_{\lambda}^{3}+\sum_{l=1}^{t} \sum_{k=1}^{\infty}(N-1) \frac{\lambda_{k}^{N+1}\left(\psi_{l}, e_{k}\right)\left(e_{k}, \varphi_{l}\right)}{\lambda^{(N+1) n}}, \\
& F_{\lambda}^{2}=\sum_{l=1}^{t} \sum_{k=1}^{\infty} \frac{\lambda_{k}^{N+2}\left(\psi_{l}, e_{k}\right)\left(e_{k}, \varphi_{l}\right)}{\lambda^{N n}\left(\lambda_{k}-\lambda^{n}\right)^{2}}, \\
& F_{\lambda}^{1}=-\sum_{l=1}^{t} \sum_{k=1}^{\infty} \frac{\lambda_{k}^{N+2}\left(\psi_{l}, e_{k}\right)\left(e_{k}, \varphi_{l}\right)}{\lambda^{(N+1) n}\left(\lambda_{k}-\lambda^{n}\right)}, \quad F_{\lambda}^{3}=\sum_{l=1}^{t} \sum_{k=1}^{\infty}(N-1) \frac{\lambda_{k}^{N+2}\left(\psi_{l}, e_{k}\right)\left(e_{k}, \varphi_{l}\right)}{\lambda^{(N+1) n}\left(\lambda_{k}-\lambda^{n}\right)} .
\end{aligned}
$$

Following the same lines as in proof of Lemma 3 , it is easy to show that $\widetilde{F}_{\lambda}=o\left(\lambda^{-N n}\right)$ as $\nu \rightarrow \infty$ uniformly in $\arg \lambda$ for $\psi_{l} \in \mathfrak{D}\left(T^{-(N+2)}\right), l=1,2, \ldots, t$, and $\lambda \in \Gamma_{\nu}$.

Formulae (20) have been obtained for $N \geqslant 2$. As $N=0,1$, by straightforward calculations we get

$$
\operatorname{Tr}\left(R_{\lambda}^{2} P\right)=o\left(\lambda^{-n}\right) \quad \nu \rightarrow \infty, \quad \lambda \in \Gamma_{\nu} .
$$

Let $k \geqslant 2$. As above, we have

$$
\operatorname{Tr}\left(Q_{\lambda}^{k}\right)=\sum_{\substack{l_{1}, l_{2}, \ldots, l_{k} \\ 0 \leqslant l_{j} \leqslant n_{j}}}\left\{\prod_{j=1}^{k-1}\left(R_{\lambda} \psi_{l_{j+1}}^{j+1}, \varphi_{l_{j}}^{j}\right)\right\}\left(R_{\lambda}^{2} \psi_{l_{1}}^{1}, \varphi_{l_{k}}^{k}\right) .
$$

Suppose that smoothness condition (17) holds true for $N \geqslant 2$. In this case we can apply formulae (15) and 20) to operator-functions $\left(\bullet, \varphi_{l_{j}}^{j}\right) R_{\lambda} \psi_{l_{j+1}}^{j+1}$ and $\left(\bullet, \varphi_{l_{k}}^{k}\right) R_{\lambda}^{2} \psi_{l_{1}}^{1}$, respectively, 
Substituting the corresponding expressions for $\left(R_{\lambda} \psi_{l_{j+1}}^{j+1}, \varphi_{l_{j}}^{j}\right)$ and $\left(R_{\lambda}^{2} \psi_{l_{1}}^{1}, \varphi_{l_{k}}^{k}\right)$ into the right hand side of identity (23), we obtain

$$
\begin{aligned}
\operatorname{Tr}\left(Q_{\lambda}^{k}\right)= & \sum_{\substack{l_{1}, l_{2}, \ldots, l_{k} \\
0 \leqslant l_{j} \leqslant n_{j}}} \prod_{j=1}^{k-1}\left\{-\sum_{p=1}^{N+1} \frac{\left(T^{-p} \psi_{l_{j+1}}^{j+1}, \varphi_{l_{j}}^{j}\right)}{\lambda^{n p}}\right\}\left\{\sum_{p=2}^{N}(p-1) \frac{\left(T^{-p} \psi_{l_{1}}^{1}, \varphi_{l_{k}}^{k}\right)}{\lambda^{n p}}\right\}+o\left(\lambda^{-n(N+k-1)}\right) \\
= & \sum_{\substack{l_{1}, l_{2}, \ldots, l_{k} \\
0 \leqslant l_{j} \leqslant n_{j}}}(-1)^{k-1} \sum_{\substack{p_{1}+p_{2}+\ldots+p_{k}=M \\
p_{1} \geqslant 2 ; p_{2}, \ldots, p_{k} \geqslant 1}}\left(p_{1}-1\right) \lambda^{-M n}\left\{\prod_{j=1}^{k-1}\left(T^{-p_{j+1}} \psi_{l_{j+1}}^{j+1}, \varphi_{l_{j}}^{j}\right)\right\}\left(T^{-p_{1}} \psi_{l_{1}}^{1}, \varphi_{l_{k}}^{k}\right) \\
& +G_{\lambda}^{M}+o\left(\lambda^{-n(N+k-1)}\right),
\end{aligned}
$$

where natural $M$ satisfies the restriction $k+1 \leqslant M \leqslant N+k-1$. Symbol $G_{\lambda}^{M}$ stands for the sum of all the terms with $p_{1}+p_{2}+\ldots+p_{k} \neq M$. These relations yield

$$
\begin{aligned}
\lim _{\nu \rightarrow \infty} & \frac{1}{2 \pi i} \oint_{\Gamma_{\nu}} \lambda^{M n-1} \operatorname{Tr}\left(Q_{\lambda}^{k}\right) d \lambda \\
& =\sum_{\substack{l_{1}, l_{2}, \ldots, l_{k} \\
0 \leqslant l_{j} \leqslant n_{j}}}(-1)^{k-1} \sum_{\substack{p_{1}+p_{2}+\ldots+p_{k}=M \\
p_{1} \geqslant 2 ; p_{2}, \ldots, p_{k} \geqslant 1}}\left(p_{1}-1\right)\left\{\prod_{j=1}^{k-1}\left(T^{-p_{j+1}} \psi_{l_{j+1}}^{j+1}, \varphi_{l_{j}}^{j}\right)\right\}\left(T^{-p_{1}} \psi_{l_{1}}^{1}, \varphi_{l_{k}}^{k}\right) \\
& =(-1)^{k-1} \sum_{\substack{p_{1}+p_{2}+\ldots+p_{k}=M \\
p_{1} \geqslant 2 ; p_{2}, \ldots, p_{k} \geqslant 1}}\left(p_{1}-1\right) \operatorname{Tr}\left(T^{-p_{1}} P_{1} \cdots T^{-p_{k}} P_{k}\right) .
\end{aligned}
$$

Let $k=1$. The smoothness condition is $\psi_{l} \in \mathfrak{D}\left(T^{-(N+2)}\right), l=1,2, \ldots, t, N \geqslant 1$. By (20)) we obtain

$$
\operatorname{Tr}\left(R_{\lambda}^{2} P\right)=\sum_{l=1}^{t} \sum_{j=2}^{N+1}(j-1) \frac{\left(T^{-j} \psi_{l}, \varphi_{l}\right)}{\lambda^{n j}}+\sum_{l=1}^{t} \sum_{k=1}^{\infty} \frac{\lambda_{k}^{N+2}\left(\psi_{l}, e_{k}\right)\left(e_{k}, \varphi_{l}\right)}{\lambda^{N n}\left(\lambda_{k}-\lambda^{n}\right)^{2}}+o\left(\lambda^{-(N+1) n}\right) .
$$

We consider the function

$$
f(\lambda)=\frac{\lambda^{s-N n}}{\left(\lambda_{k}-\lambda^{n}\right)^{2}}
$$

where $s$ is a natural number, $s \geqslant N n$. Function $f(\lambda)$ has poles at the points $\lambda=\eta_{k_{l}}: \eta_{k_{l}}^{n}=\lambda_{k}$, $l=1, \ldots, n$. The residues at these poles can be easily calculated:

$$
\operatorname{Res}_{\eta_{k_{l}}} f(\lambda)=\frac{s-(N+1) n+1}{n^{2}} \eta_{k_{l}}^{s-(N+2) n+1} \quad l=1, \ldots, n .
$$

Hence, it is easy to see that

$$
\sum_{l=1}^{n} \operatorname{Res}_{\eta_{k_{l}}} f(\lambda)=0
$$

if either $s+1=(N+1) n$ or $s+1$ is not a multiple of $n$. In the former case $\operatorname{Res}_{\eta_{k_{l}}} f(\lambda)$ vanishes for each $l=1, \ldots, n$, while in the latter

$$
\sum_{l=1}^{n} \operatorname{Res}_{\eta_{k_{l}}} f(\lambda)=\frac{s-(N+1) n+1}{n^{2}} \sum_{l=1}^{n} \eta_{k_{l}}^{s-(N+2) n+1}=0,
$$

i.e., $\eta_{k_{1}}^{s}+\eta_{k_{2}}^{s}+\ldots+\eta_{k_{n}}^{s}$ for each natural $s$ not being a multiple of $n$.

The above arguments imply that

$$
\lim _{\nu \rightarrow \infty} \frac{1}{2 \pi i} \oint_{\Gamma_{\nu}} \lambda^{M n-1} \operatorname{Tr}\left(R_{\lambda}^{2} P\right) d \lambda=N \cdot \operatorname{Tr}\left(T^{-(N+1)} P\right) .
$$


as $N \geqslant 1$ and $M=N+1$.

Lemma 6. Suppose that we are given finite-dimensional operators $P_{1}, P_{2}, \ldots, P_{k}$. Assume that smoothness condition (17) is satisfied. Then

1. If $k \geqslant 2$ and $N \geqslant 2, N$ is the integer in the smoothness condition (17), then for each integer $M: k+1 \leqslant M \leqslant N+k-1$ identity (25) holds true. As $M \leqslant k$, the left hand side in (25) vanishes. If in the left hand side in (25) we replace $M n$ by an integer $s: s<(N+k-1) n$ not being a multiple of $n$, then the left hand side in (25) vanishes, too.

2. If $k=1$, for $N \geqslant 1$, where $N$ is the integer in condition (17), the identity (26) holds true. If in (26) instead of $(N+1) n$ we substitute $s: s<(N+1) n$ not being a multiple of $n$, then the left hand side in (26) vanishes.

3. If $N=0$ in $(17)$, then

for $k \geqslant 1$.

$$
\lim _{\nu \rightarrow \infty} \oint_{\Gamma_{\nu}} \lambda^{n-1} \operatorname{Tr}\left(Q_{\lambda}^{k}\right) d \lambda=0
$$

The latter identity follows from relation $(22)$.

\section{Regularized trace FORMulae}

In what follows we assume that natural parameter $s$ takes values from $N n+1$ to $(N+$ 1) $n$, where $N \geqslant 0$ is integer. Moreover, finite-dimensional operators $P_{0}, P_{1}, \ldots, P_{n-1}$ satisfy smoothness condition (17).

5.1. Calculation of $\lim _{\nu \rightarrow \infty} J_{1}^{\nu}(s)$. We have

$$
J_{1}^{\nu}(s)=\sum_{j=1}^{n-1} \frac{j}{2 \pi i} \oint_{\Gamma_{\nu}} \lambda^{j+s-1} \operatorname{Tr}\left(R_{\lambda} P_{j}\right) d \lambda .
$$

It follows from 5 for $k=1$ and $M n=j+s=(N+1) n$ that

Lemma 7. Let $N n+1 \leqslant s \leqslant(N+1) n$ with integer $N \geqslant 0$.

1. The identity

$$
G_{1}(s) \stackrel{\text { def }}{=} \lim _{\nu \rightarrow \infty} J_{1}^{\nu}(s)=-j_{s} \sum_{l=1}^{n_{j_{s}}}\left(T^{-(N+1)} \psi_{l}^{j_{s}}, \varphi_{l}^{j_{s}}\right)=-j_{s} \operatorname{Tr}\left(T^{-(N+1)} P_{j_{s}}\right)
$$

holds true, where $j_{s}=(N+1) n-s$.

2. If $s=t n$ for each integer $t \geqslant 0$, then $G_{1}(s)=\lim _{\nu \rightarrow \infty} J_{1}^{\nu}(s)=0$.

5.2. Calculation of $\lim _{\nu \rightarrow \infty} J_{2}^{\nu}(s)$. We have

$$
J_{1}^{\nu}(s)=\sum_{j=1}^{n-1} \sum_{k=1}^{\infty} \frac{j}{2 \pi i} \oint_{\Gamma_{\nu}} \lambda^{j+s-1} \operatorname{Tr}\left(R_{\lambda} P_{j}\left(R_{\lambda} P_{0}\right)^{k}\right) d \lambda .
$$

Lemma 5 implies

Lemma 8. Let $N n+1 \leqslant s \leqslant(N+1) n$ with integer $N \geqslant 0$.

1. The identity

$$
G_{2}(s) \stackrel{\text { def }}{=} \lim _{\nu \rightarrow \infty} J_{2}^{\nu}(s)=j_{s} \sum_{k=1}^{N}(-1)^{k+1} \sum_{\substack{p_{0}+p_{1}+\cdots+p_{k}=N+1 \\ p_{j} \geqslant 0}} \operatorname{Tr}\left(T^{-p_{0}} P_{j_{s}} T^{-p_{1}} P_{0} \cdots T^{-p_{k}} P_{0}\right)
$$


holds true, where $j_{s}=(N+1) n-s$.

2. As $s=t n, t \geqslant 0$ is integer, we have $G_{2}(s)=\lim _{\nu \rightarrow \infty} J_{2}^{\nu}(s)=0$.

5.3. Вычисление $\lim _{\nu \rightarrow \infty} J_{3}^{\nu}(s)$. We have

$$
J_{3}^{\nu}(s)=\sum_{j=1}^{n-1} \sum_{k=1}^{\infty} \sum_{m=1}^{k(n-1)} \frac{j}{2 \pi i} \oint_{\Gamma_{\nu}} \lambda^{j+s+m-1} \operatorname{Tr}\left(R_{\lambda} P_{j} \sum_{m} P_{\alpha_{1} \ldots \alpha_{k}}\right) d \lambda .
$$

It follows from 18$)$ that

$$
\begin{gathered}
1 \leqslant k \leqslant j+s-n . \\
j_{s} \leqslant j \leqslant n-1, \quad \text { where } j_{s} \stackrel{\text { def }}{=} \max \{1, n-s+1\} .
\end{gathered}
$$

The limit of the integral in the expression $J_{3}^{\nu}(s)$ is non-zero if for each fixed index $k$ we have $(k+t+1) n=j+s+m$ for some integer $t \geqslant 0$. It is easy to obtain

$$
t_{0} \leqslant t \leqslant t_{1}, \quad t_{0}=\overline{[(j+s+1) / n-k-1]}, \quad t_{1}=[(j+s-k-n) / n],
$$

where $\overline{[a]}$ stands for the least integer greater or equal $a$.

By Lemma 5 we obtain the following result.

Lemma 9. 1. The identities

$$
\begin{aligned}
& J_{3}(j, k, t, s) \stackrel{\text { def }}{=} \lim _{\nu \rightarrow \infty} \frac{1}{2 \pi i} \oint_{\Gamma_{\nu}} \lambda^{j+s+m-1} \operatorname{Tr}\left(R_{\lambda} P_{j} \sum_{m} P_{\alpha_{1} \ldots \alpha_{k}}\right) d \lambda \\
& =(-1)^{k+1} \sum_{\substack{p_{0}+p_{1}+\cdots+p_{k}=k+t+1 \\
p_{l} \geqslant 0 ; l=0,1, \ldots, k}} \sum_{\substack{\alpha_{1}+\cdots+\alpha_{k}=(k+t+1)-(j+s) \\
\alpha_{l} \geqslant 0 ; l=1, \ldots, k}} \operatorname{Tr}\left(T^{-p_{0}} P_{j} T^{-p_{1}} P_{\alpha_{1}} \cdots T^{-p_{k}} P_{\alpha_{k}}\right) \\
& G_{3}(s) \stackrel{\text { def }}{=} \lim _{\nu \rightarrow \infty} J_{3}^{\nu}(s)=\sum_{j=j_{s}}^{n-1} j \sum_{k=1}^{j+s-n} \sum_{t=t_{0}}^{t_{1}} J_{3}(j, k, t, s)
\end{aligned}
$$

hold true.

2. As $s=0,1$, the identity $\lim _{\nu \rightarrow \infty} J_{3}^{\nu}(s)=0$ holds true. It follows from the fact that the set of indices $j$ defined by inequlaities (28) is empty for $s=0,1$.

5.4. Calculation of $\lim _{\nu \rightarrow \infty} J_{4}^{\nu}(s)$. We have

$$
J_{4}^{\nu}(s)=\sum_{k=1}^{\infty} \frac{n}{2 \pi i} \oint_{\Gamma_{\nu}} \lambda^{n+s-1} \operatorname{Tr}\left(T R_{\lambda}\left(R_{\lambda} P_{0}\right)^{k}\right) d \lambda .
$$

We employ the obvious identity $T R_{\lambda}=\lambda^{-n} R_{\lambda}-\lambda^{-n} E$ and substitute it into the formula for $J_{4}^{\nu}(s)$ to obtain

$$
J_{4}^{\nu}(s)=n \sum_{k=1}^{\infty} \frac{1}{2 \pi i} \oint_{\Gamma_{\nu}} \lambda^{s-1} \operatorname{Tr}\left(R_{\lambda}\left(R_{\lambda} P_{0}\right)^{k}\right) d \lambda-n \sum_{k=1}^{\infty} \frac{1}{2 \pi i} \oint_{\Gamma_{\nu}} \lambda^{s-1} \operatorname{Tr}\left(\left(R_{\lambda} P_{0}\right)^{k}\right) d \lambda .
$$

Applying Lemma 6 to the first term and Lemma 5 to the second term, we arrive at 
Lemma 10. 1. The identities

$$
\begin{aligned}
G_{4}^{1}(s) & \stackrel{\text { def }}{=} \lim _{\nu \rightarrow \infty} n \sum_{k=1}^{\infty} \frac{1}{2 \pi i} \oint_{\Gamma_{\nu}} \lambda^{s-1} \operatorname{Tr}\left(R_{\lambda}\left(R_{\lambda} P_{0}\right)^{k}\right) d \lambda \\
& =n \sum_{k=1}^{N}(-1)^{k+1} \sum_{\substack{p_{1}+p_{2}+\cdots+p_{k}=N+1 \\
p_{l} \geqslant 2 ; p_{2}, \ldots, p_{k} \geqslant 1}}\left(p_{1}-1\right) \operatorname{Tr}\left(T^{-p_{1}} P_{0} T^{-p_{2}} P_{0} \cdots T^{-p_{k}} P_{0}\right) \\
G_{4}^{2}(s) & \stackrel{\text { def }}{=}-\lim _{\nu \rightarrow \infty} n \sum_{k=1}^{\infty} \frac{1}{2 \pi i} \oint_{\Gamma_{\nu}} \lambda^{s-1} \operatorname{Tr}\left(\left(R_{\lambda} P_{0}\right)^{k}\right) d \lambda \\
& =n \sum_{k=1}^{N+1}(-1)^{k+1} \sum_{\substack{p_{1}+p_{2}+\cdots+p_{k}=N+1 \\
p_{l}, p_{2}, \ldots, p_{k} \geqslant 1}} \operatorname{Tr}\left(T^{-p_{1}} P_{0} T^{-p_{2}} P_{0} \cdots T^{-p_{k}} P_{0}\right)
\end{aligned}
$$

hold true, i.e., $\lim _{\nu \rightarrow \infty} J_{4}^{\nu}(s)=G_{4}^{1}(s)+G_{4}^{2}(s)$.

2. If parameter $s \in \mathbb{N}$ is not a multiple of $n$, in particular, as $s=0,1, \ldots, n-1$, then $\lim _{\nu \rightarrow \infty} J_{4}^{\nu}(s)=0$.

5.5. Calculation of $\lim _{\nu \rightarrow \infty} J_{5}^{\nu}(s)$. We have

$$
J_{5}^{\nu}(s)=\sum_{k=1}^{\infty} \sum_{m=1}^{k(n-1)} \frac{n}{2 \pi i} \oint_{\Gamma_{\nu}} \lambda^{m+n+s-1} \operatorname{Tr}\left(R_{\lambda} T \sum_{m} P_{\alpha_{1} \ldots \alpha_{k}}\right) d \lambda .
$$

We apply the identity $T R_{\lambda}=\lambda^{-n} R_{\lambda}-\lambda^{-n} E$ to obtain

$$
\begin{aligned}
J_{5}^{\nu}(s)= & n \sum_{k=1}^{\infty} \sum_{m=1}^{k(n-1)} \frac{1}{2 \pi i} \oint_{\Gamma_{\nu}} \lambda^{m+s-1} \operatorname{Tr}\left(R_{\lambda} \sum_{m} P_{\alpha_{1} \ldots \alpha_{k}}\right) d \lambda \\
& -n \sum_{k=1}^{\infty} \sum_{m=1}^{k(n-1)} \frac{1}{2 \pi i} \oint_{\Gamma_{\nu}} \lambda^{m+s-1} \operatorname{Tr}\left(\sum_{m} P_{\alpha_{1} \ldots \alpha_{k}}\right) d \lambda .
\end{aligned}
$$

We denote by $J_{5}^{1 \nu}(s)$ and $J_{5}^{2 \nu}(s)$ the terms in the right hand side of the latter formula, respectively.

By (6) we get the range of index $k$ :

$$
1 \leqslant k \leqslant s-n \text {. }
$$

As in the proof of Lemma 10 , we assume $(t+k+1) n=m+s$ for integer $t \geqslant 0$, where

$$
\widetilde{t}_{0} \stackrel{\text { def }}{=} \overline{[(s-(k+1) n+1) / n]} \leqslant t \leqslant[(s-k-n) / n] \stackrel{\text { def }}{=} \widetilde{t}_{1},
$$

Applying Lemma 6, we obtain

$$
\begin{aligned}
& G_{5}^{1}(s) \stackrel{\text { def }}{=} \lim _{\nu \rightarrow \infty} J_{5}^{1 \nu}(s)=n \sum_{k=1}^{s-n} \sum_{t=\widetilde{t}_{0}}^{\widetilde{t}_{1}}(-1)^{k+1} \\
& \cdot \sum_{\substack{p_{1}+\ldots+p_{k}=k+t+1 \\
p_{l} \geqslant 2, p_{2}, \ldots, p_{k} \geqslant 1}}\left(p_{1}-1\right) \sum_{\substack{\alpha_{1}+\ldots+\alpha_{k}=(k+t+1) n-s \\
0 \leqslant \alpha_{l} \leqslant n-1 ; l=1, \ldots, k}} \operatorname{Tr}\left(T^{-p_{1}} P_{\alpha_{1}} \cdots T^{-p_{k}} P_{\alpha_{k}}\right) . \\
&
\end{aligned}
$$

To calculate $\lim _{\nu \rightarrow \infty} J_{5}^{2 \nu}(s)$, we reproduce the same calculations but at the last step we apply Lemma 5 . 
We have $1 \leqslant k \leqslant s$. For each $k$, the identity holds $m+s=(k+\mu) n$ for some integer $\mu \geqslant 0$, where

$$
1+s \leqslant(k+\mu) n \leqslant k(n-1)+s, \quad \widetilde{\mu}_{0} \stackrel{\text { def }}{=} \overline{[(s-n k+1) / n]} \leqslant \mu \leqslant[(s-k) / n] \stackrel{\text { def }}{=} \widetilde{\mu}_{1} .
$$

Applying Lemma 5, in view of (35) we get

$$
\begin{aligned}
& G_{5}^{2}(s) \stackrel{\text { def }}{=} \lim _{\nu \rightarrow \infty} J_{5}^{2 \nu}(s)=n \sum_{k=1}^{s} \sum_{\mu=\widetilde{\mu}_{0}}^{\widetilde{\mu}_{1}}(-1)^{k-1} \\
& \text { - } \sum_{\substack{p_{1}+\ldots+p_{k}=k+\mu \\
p_{l}, p_{2}, \ldots, p_{k} \geqslant 1}} \sum_{\substack{\alpha_{1}+\ldots+\alpha_{k}=(k+\mu) n-s \\
0 \leqslant \alpha_{l} \leqslant n-1 ; l=1, \ldots, k}} \operatorname{Tr}\left(T^{-p_{1}} P_{\alpha_{1}} \cdots T^{-p_{k}} P_{\alpha_{k}}\right) \text {. }
\end{aligned}
$$

Lemma 11. 1. The identity $\lim J_{5}^{\nu}(s)=G_{5}^{1}(s)+G_{5}^{2}(s)$ holds true, where $G_{5}^{1}(s)$ and $G_{5}^{2}(s)$ are determined by identities $\sqrt{(34)}$ and $(36)$.

2. $\lim _{\nu \rightarrow \infty} J_{5}^{\nu}(0)=0$. It can be proven easily in the same way as second items in previous lemmata 710 .

Corollary 3. Now we can prove Lemma 4 in accordance with which function $F(s)$ defined by formula (11) satisfies $F(0)=0$. But in view of (13) it is implied by the identity $\lim _{\nu \rightarrow \infty} J_{k}^{\nu}(0)=0$ $\forall k=1,2,3,4,5$. These identities were justified in Lemmata 711 .

We summarize all the above arguments in the following theorem.

Theorem 1. Suppose that we are given operator pencil

$$
L_{\lambda}=E-P_{0}-\lambda P_{1} \cdots \lambda^{n-1} P_{n-1}-\lambda^{n} T
$$

in separable Hilbert space $\mathfrak{H}$, where operators $P_{0}, P_{1}, \ldots, P_{n-1}$ are finite-dimensional and read as $P_{j}=\sum_{l=1}^{n_{j}}\left(\bullet, \varphi_{l}^{j}\right) \psi_{l}^{j}$, while $T$ is an injective self-adjoint compact operator in $\mathfrak{H}$. Suppose that the counting function of the eigenvalues of pencil $T_{\lambda}=E-\lambda T$ satisfies "sparseness" condition (6).

Suppose that $s \in \mathbb{N} \cap[N n+1,(N+1) n]$ with integer $N \geqslant 0$. If $[17)$ holds, there exists a monotonous sequence of the natural series $\left\{N_{\nu}\right\}_{\nu=m_{0}}^{\infty}$ satisfying the regularized trace formula

$$
\lim _{\nu \rightarrow \infty} \sum_{m=1}^{N_{\nu}}\left(\mu_{m}^{s}-\eta_{m}^{s}-c_{m}(s)\right)=F(s),
$$

where $\mu_{m}$ and $\eta_{m}$ are the eigenvalues of pencils $L_{\lambda}$ and $T_{\lambda^{n}}$, respectively taken counting multiplicities, $c_{m}(s)=0$, and

$$
F(s)=-G_{1}(s)-G_{2}(s)-G_{3}(s)-G_{4}^{1}(s)-G_{4}^{2}(s)-G_{5}^{1}(s)-G_{5}^{2}(s),
$$

where values $G_{j}^{i}(s)$ are determined in Lemmata 711 .

\section{EXAMPLE}

We consider the second order pencil $L_{\lambda}=E-P_{0}-\lambda P_{1}-\lambda^{2} T$. Assuming the hypothesis of Theorem 1, let us write down regularized trace formulae of first, second, and third orders. Quantities $G_{j}^{l}(s)$ defined in Theorem 1 read as

1. $G_{1}(1)=-\operatorname{Tr}\left(T^{-1} P_{1}\right), G_{2}(1)=G_{3}(1)=G_{4}^{1}(1)=G_{4}^{2}(1)=G_{5}^{1}(1)=0, G_{5}^{2}(1)=2 \operatorname{Tr}\left(T^{-1} P_{1}\right)$;

2. $G_{1}(2)=G_{2}(2)=G_{4}^{1}(2)=G_{5}^{1}(2)=0, G_{3}(2)=\operatorname{Tr}\left(\left(T^{-1} P_{1}\right)^{2}\right), G_{4}^{2}(2)=2 \operatorname{Tr}\left(T^{-1} P_{0}\right)$, $G_{5}^{2}(2)=-2 \operatorname{Tr}\left(\left(T^{-1} P_{1}\right)^{2}\right)$

3. $G_{1}(3)=-\operatorname{Tr}\left(\left(T^{-2} P_{1}\right)^{2}\right), G_{2}(3)=\operatorname{Tr}\left(T^{-1} P_{1} T^{-1} P_{0}\right), G_{3}(3)=-\operatorname{Tr}\left(\left(T^{-1} P_{1}\right)^{3}\right), G_{4}^{1}(3)=$ $G_{4}^{2}(3)=0, G_{5}^{1}(3)=2 \operatorname{Tr}\left(T^{-2} P_{1}\right), G_{5}^{2}(1)=2 \operatorname{Tr}\left(T^{-2} P_{1}\right)-4 \operatorname{Tr}\left(T^{-1} P_{1} T^{-1} P_{0}\right)+2 \operatorname{Tr}\left(\left(T^{-1} P_{1}\right)^{3}\right)$. 
The first group of formulae by Theorem 1 implies

$$
\lim _{\nu \rightarrow \infty} \sum_{m=1}^{N_{\nu}}\left(\mu_{m}-\eta_{m}\right)=-\operatorname{Tr}\left(T^{-1} P_{1}\right) .
$$

The second group of formulae yields

$$
\lim _{\nu \rightarrow \infty} \sum_{m=1}^{N_{\nu}}\left(\mu_{m}^{2}-\eta_{m}^{2}\right)=-\operatorname{Tr}\left(\left(T^{-1} P_{1}\right)^{2}\right)-2 \operatorname{Tr}\left(T^{-1} P_{0}\right) .
$$

It follows from the third group of formulae:

$$
\lim _{\nu \rightarrow \infty} \sum_{m=1}^{N_{\nu}}\left(\mu_{m}^{3}-\eta_{m}^{3}\right)=-\operatorname{Tr}\left(\left(T^{-1} P_{1}\right)^{3}\right)-3 \operatorname{Tr}\left(T^{-2} P_{1}\right)+3 \operatorname{Tr}\left(T^{-1} P_{1} T^{-1} P_{0}\right)
$$

We apply the obtained result to the Sturm-Liouville problem for a loaded equation. We consider the boundary value problem

$$
\begin{aligned}
& -y^{\prime \prime}(x)+q(x) y(x)-a(x) y\left(x_{0}\right)-\lambda b(x) y\left(x_{1}\right)-\lambda^{2} y(x)=0, \quad 0<x<\pi, \\
& y(0)=y(\pi)=0, \quad x_{0}, x_{1} \in(0, \pi) .
\end{aligned}
$$

By $A$ we denote the self-adjoint operator in $L_{2}(0, \pi): A y(x)=-y^{\prime \prime}(x)+q(x) y(x), D(A)=\{y \in$ $\left.W_{2}^{2}(0, \pi): y(0)=y(\pi)\right\}$.

Let $G(x, \xi)$ be the Green function of operator $A$. Then we have the identities

$$
y\left(x_{0}\right) a(x)=a(x) \int_{0}^{\pi} G\left(x_{0}, \xi\right) A y(\xi) d \xi, \quad y\left(x_{1}\right) b(x)=b(x) \int_{0}^{\pi} G\left(x_{1}, \xi\right) A y(\xi) d \xi
$$

Thus, the boundary value problem generates operator pencil $N_{\lambda}=A-Q_{0}-\lambda Q_{1}-\lambda^{2} E$, where

$$
Q_{0} y(x)=\int_{0}^{\pi} a(x) G\left(x_{0}, \xi\right) A y(\xi) d \xi, \quad Q_{1} y(x)=\int_{0}^{\pi} b(x) G\left(x_{1}, \xi\right) A y(\xi) d \xi .
$$

Pencil $L_{\lambda}=N_{\lambda} A^{-1}$ satisfies the hypothesis of Theorem 1. Thus, if $a(x), b(x) \in D\left(A^{2}\right)$, in accordance with formulae (37), (38)

$$
\lim _{\nu \rightarrow \infty} \sum_{m=1}^{N_{\nu}}\left(\mu_{m}-\eta_{m}\right)=-b\left(x_{1}\right), \quad \lim _{\nu \rightarrow \infty} \sum_{m=1}^{N_{\nu}}\left(\mu_{m}^{2}-\eta_{m}^{2}\right)=b^{2}\left(x_{1}\right)-2 a\left(x_{0}\right) .
$$

If $a(x), b(x) \in D\left(A^{3}\right)$, formula 39$)$ implies

$$
\lim _{\nu \rightarrow \infty} \sum_{m=1}^{N_{\nu}}\left(\mu_{m}^{3}-\eta_{m}^{3}\right)=-b^{3}\left(x_{1}\right)-3\left[-b^{\prime \prime}\left(x_{1}\right) q\left(x_{1}\right) b\left(x_{1}\right)\right]+3 a\left(x_{1}\right) b\left(x_{0}\right) .
$$

\section{BIBLIOGRAPHY}

1. Yu.A. Anokhin, A.B. Torstko, L.Yu. Dameshek, G.N. Konstantinov. Mathematical models and control methods of large-scale water object. Nauka, Novosibirsk (1987). (in Russian).

2. A.M. Nakhushev. Loaded equations and their applications // Differ. Uravn. 19:1, 86-94 (1983). [Differ. Equat. 19:1, 74-81 (1983).]

3. V.A. Sadovnichii, V.A. Lyubishkin. Finite-dimensional perturbations of discrete operators and formulas for traces // Funkt. Anal. Pril. 20:3, 55-65 (1986). [Funct. Anal. Appl. 20:3, 214-223 (1986).]

4. V.A. Lyubishkin, I.D. Tsopanov. On new trace formulae for operators with discrete spectrum // Vestn. Mosk. Univ. Ser. I. 6, 22-25 (1987). (in Russian).

5. V.A. Sadovnichij, V.V. Dubrovskij, V.A. Lyubishkin. Traces of discrete operators // Dokl. Akad. Nauk SSSR. 264:4, 830-832 (1982). [Sov. Math., Dokl. 25, 759-761 (1982).] 
6. V.A. Sadovnichij. Operator theory. Vysshaya shkola, Moscow (1999). (in Russian).

7. Z.Yu. Fazullin. Non-nuclear perturbation of discrete operators and trace formulae. Habilitation Thesis. Institute of Mathematics CC USC RAS, Ufa (2006). (in Russian).

8. V.A. Sadovnichii, V.E. Podol'skii. Traces of operators // Uspekhi Matem. Nauk. 61:5(371), 89-156 (2006). [Russ. Math. Surv. 61:5, 885-953 (2006).]

9. Yu.V. Matveev. Spectral properties of differential-functional operators // PhD Thesis. Lomonosov Moscow State University, Moscow (1986). (in Russian).

10. N.A. Kulesko, Yu.A. Palant. To E.I. Sigal theorem on trace of operator pencil // Matem. Issled. 6:2, 150-152 (1971). (in Russian).

11. N.A. Kulesko. On traces of polynomial operator pencil // Funkts. Anal. 25, 87-91 (1985). (in Russian).

12. E.I. Sigal. On trace of operator pencil // Matem. Issled. 4:2, 148-151 (1969). (in Russian).

13. M.I. Gil'. Sums of characteristic values of compact polynomial operator pencils // J. Math. Anal. Appl. 338:2, 1469-1476 (2008).

14. H. König. A trace theorem and a linearization method for operator polynomials // Int. Equat. Oper. Theory. 5:1, 828-849 (1982).

15. I.C. Gohberg, M.G. Krein. Introduction to the theory of linear nonselfadjoint operators. Nauka, Moscow (1965). [Trans. Math. Monographs. 18. Amer. Math. Soc., Providence, RI (1969).]

16. M.V. Keldysh. On the completeness of the eigenfunctions of some classes of non-selfadjoint linear operators // Uspekhi Matem. Nauk. 26:4, 15-41 (1971). [26:4, 15-44 (1971).]

17. I.D. Tsopanov. General formulas for regularized traces of integrodifferential operators Vladikavkaz. Matem. Zhurn. 9:4, 32-48 (2007). (in Russian).

Igor' Dzastemirovich Tsopanov,

South Mathematical Institute,

Russian Academy of Sciences,

Markus str., 22,

362027, Vladikavkaz, Russia

Vladikavkaz Administration Institute,

Borodinskaya str., 14,

362025, Vladikavkaz, Russia

E-mail: 55tsopanovig@gmail.com 